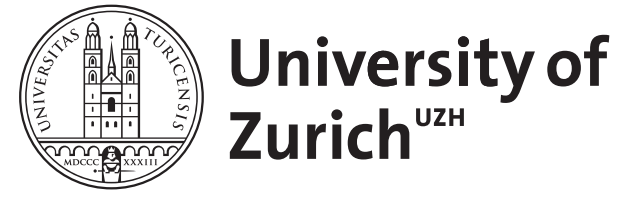

\title{
Feline Acromegaly
}

Reusch, Claudia E

DOI: https://doi.org/10.1053/j.tcam.2012.05.004

Posted at the Zurich Open Repository and Archive, University of Zurich

ZORA URL: https://doi.org/10.5167/uzh-127471

Conference or Workshop Item

Originally published at:

Reusch, Claudia E (2016). Feline Acromegaly. In: ESVE, Summer School of Veterinary Endocrinology, Bologna, Italy, 26 June 2016 - 1 July 2016, s.n..

DOI: https://doi.org/10.1053/j.tcam.2012.05.004 
Feline Acromegaly

Claudia E. Reusch, Dipl ECVIM-CA

\section{Introduction and prevalence of feline acromegaly}

The term acromegaly derives from the Greek words akron «extreme or extremity» and megas „big, large." The term is used to describe the result of Growth Hormone (GH) excess in adults, i.e. after the closure of the epiphyseal plates, $\mathrm{GH}$ excess prior to epiphyseal plate closure leads to linear growth and large stature, termed gigantism.

In 1976, Gembardt and Loppnow described the first two cats with diabetes mellitus and acidophilic pituitary adenomas. Although $\mathrm{GH}$ was not measured, the authors proposed that $\mathrm{GH}$ excess from the tumors was responsible for the diabetes. During the following 15 years further cases of acromegaly/hypersomatotropism (HS) were only published sporadically (Eigenmann et al 1984, Middleton et al 1985, Lichtensteiger et al 1986, Heinrichs et al 1989, Morrison et al 1989). The first case series of HS was published by Mark Peterson et al in 1990. The authors described clinical features and results of diagnostic work-up in 14 cats, which had been diagnosed with HS between 1981 and 1988 in 7 different veterinary institutions. All 14 cats were diabetic and revealed increasing insulin needs within a few weeks to months after the start of therapy. At that time feline HS was considered to be a rare disease. The rareness started to be questioned in 2007 when two larger studies were published in the same issue of the Journal of Veterinary Internal Medicine (Berg et al 2007, Niessen et al 2007). The publications were accompanied by an editorial by Mark Peterson, entitled: Feline Acromegaly: Are we only diagnosing the tip of the iceberg? Since then the number of publications dealing with various clinical, diagnostic and therapeutical aspects of feline HS has increased substantially. It is now known that HS is not as rare as previously thought. However, the question on the true prevalence of feline HS is currently still unsolved as only very few prospective studies have been performed. Niessen et al (2007) screened blood samples which had been sent by veterinary practices throughout the UK for the measurement of fructosamine. In 59 of the 184 cats (32\%) with variable control of the diabetes IGF-1 was markedly increased (> $1000 \mathrm{ng} / \mathrm{ml}$ ). Further work-up by brain imaging and/or histopathology was possible in 18 cats and HS was confirmed in 17 of them. More recently, the same research group investigated a large cohort of cats and found that IGF-1 was elevated $>1000 \mathrm{ng} / \mathrm{ml}$ in 334 of 1222 diabetic cats (27.3\%). In approximately half of the cats the clinician strongly suspected HS or thought that HS may be present. A subset of 63 of the 334 cats underwent pituitary imaging and 56/63 had a pituitary tumor on CT, an additional 3 cats on MRI and one on necropsy. The authors extrapolated from those data that HS is present in approximately $25 \%$ of diabetic cats (Niessen et al 2015). We recently screened diabetic cats treated in private practices in the Netherlands and in Switzerland (cooperation with Hans Kooistra from Utrecht; Schäfer et al, submitted). The percentage of increased IGF-1 was lower than in the studies of Niessen et al $(2007,2015)$. 36/202 diabetic cats (17.8\%) had IGF-1 > $1000 \mathrm{ng} / \mathrm{ml}$, CT was possible in 5/36 and a pituitary mass was only found in one of the 5 cats. At our institution we systematically screen all cats with diabetes mellitus independent of the quality of glycemic control. Between February 2015 and February 201640 cats with diabetes were newly presented to our endocrine service. HS was diagnosed in only 3 of them (7.5\%) by means of increased IGF-1 and pituitary imaging. This is slightly lower than the percentage of previous years. We currently assume, that in our country the prevalence of HS in the diabetic cat population is $10-15 \%$. 


\section{Etiopathogenesis}

In the vast majority of cats, HS is caused by an GH-producing acidophil pituitary adenoma. Capen (2007) described densely granulated and sparsely granulated cells in the adenomas, it is, however, unknown so far if those findings represent different variants of the disease as known in humans. In the latter sparsely granulated staining cytoplasmatic $\mathrm{GH}$ adenomas are more likely to be locally invasive and grow faster than their densely granulated counterpart and they occur at younger age (Lopes 2010). By means of immunohistochemistry it has been demonstrated that the pituitary tumors in cats may either only stain positive for GH (Heinrichs et al 1989, Meij et al 2004, Meij et al 2010) or they may mainly stain positive for GH, but also for other hormones such as prolactin, ACTH, $\beta$-endorphin, FSH and TSH (Middleton et al 1985, Allgoewer et al 1998, Sharman et al 2013). Those mixed tumors, in which several hormones are synthesized within the same mass should be differentiated from so-called double adenoma. Meij et al (2004) reported the concurrent existence of a somatotroph and a corticotroph adenoma in a cat with diabetes mellitus. The clinical picture was dominated by insulin-resistant diabetes mellitus and hyperadrenocorticism, physical changes of acromegaly were not obvious. Sharman et al (2013) recently reported another case of double adenoma. The cat was referred for insulin-resistant diabetes and physical changes consistent with acromegaly, 16 months later the cat developped additional signs consistent with hyperadrenocorticism. Necrophy confirmed the presence of an acidophilic adenoma staining strongly positive for $\mathrm{GH}$, with some positivity of $\mathrm{FSH}, \mathrm{TSH}$ and $\beta$-endorphin consistent with a somatotroph adenoma, the other chromophobe adenoma stained strongly positive for $\mathrm{ACTH}, \mathrm{MSH}, \mathrm{FSH}$ and $\beta$-endorphin consistent with a plurihormonal tumor.

On rare occasions $\mathrm{GH}$ excess may not derive from an acidophilic adenoma but from acidophilic hyperplasia (Norman and Mooney 2000, Niessen et al 2007). It is possible that hyperplasia is the precursor step of adenoma, however, the possibility of a different etiology (e.g. GHRH excess) also has to be taken into account. It is important to realize, that in those cases pituitary imaging with CT/MRI may be negative.

A recent study showed that diabetic cats with acromegaly have significantly higher concentrations of organohalogenated contaminants in plasma compared to non-acromegalic diabetes cats and control cats and it was suggested, that acromegalic cats have a lower capacity to metabolize those substances. Further studies are needed to explore a possible link between feline HS and exposure to organohalogenated contaminants (Dirtu et al 2013).

In HS circulating GH concentrations are chronically increased, although secretion remains episodic. GH has direct and indirect metabolic effects, the latter are mediated through the stimulation of IGF-1 synthesis in the liver and other tissues. The growth promoting effects of increased GH and IGF-1 result in the proliferation of bone, cartilage, soft tissues and increase in size of various organs. Additionally, GH has anti-insulin actions, whereas IGF-1 enhances insulin sensitivity in hepatic and extrahepatic tissues. In HS increased IGF-1 levels are unable to counter-act the insulin resistance induced by the excessive GH concentrations (Resmini et al 2009). In normal individuals, insulin resistance is compensated by an increased insulin production from the $\beta$-cells, diabetes only develops in individuals in which $\beta$-cells fail to meet the increased demand. The majority of cats with HS suffer from diabetes mellitus. Insulin requirements may range from normal to highly increased. So far, the reason for $\beta$-cell failure in the case of HS is unknown.

\section{Signalement, clinical manifestations and clinical pathology}

$\mathrm{HS}$ is typically diagnosed in elderly cats with a mean age of $10-11$ years. Most cats are Domestic Short-Haired or Domestic Long-Haired and there appears to be a male predisposition. The earliest and most common clinical signs usually are pu/pd and polyphagia associated with $\mathrm{GH}$-induced diabetes. Polyphagia may also develop as a direct effect of the GH excess and can become extreme. Most - but not all - cats with acromegaly are diabetic. The possibility of underlying acromegaly is often times only considered after the 
diabetes becomes difficult to regulate. However, it should be noted that not all acromegalic cats with diabetes are insulin-resistant. In some of the cats a "normal" insulin dose (1-3 U/cat $\mathrm{BID}$ ) is sufficient for long-term adequate control. Even diabetic remission can occur in rare cases without treatment of the GH excess. The physical changes of HS are induced by the anabolic effects of GH and IGF-1, they have an insidious onset and progress very slowly. Manifestations of HS include enlargement of face (broadening), abdomen and paws, prognathia inferior, organomegaly, respiratory stridor, stiffness or lameness (due to degenerative arthropathy), systolic heart murmur and/or gallop rhythm and CNS signs (in case of a large pituitary tumor). Initial weight loss may be followed by weight gain, a cat with difficult to regulate diabetes and weight gain should always alert the clinician to consider the presence of HS. Of note, many cats with HS are unremarkable during physical examination or reveal only very subtle signs (Reusch 2015).

Cardiomyopathy may either be the result of GH excess or the latter worsens a pre-existing cardiomyopathy. ECG is usually unremarkable (Peterson et al 1990, Niessen et al 2007). Blood pressure is either normal or slightly elevated. Echocardiographic findings include generalized or focal (septal, basal, free wall) left ventricular hypertrophy with or without left atrial dilatation. In some cats, right heart disease with right-sided heart failure may predominate. Echocardiography, however, can also be unremarkable (Myers et al 2014, Reusch 2015).

The often poorly controlled diabetes is responsible for most of the abnormal findings on CBC, chemistry profile and urinalysis. Hyperproteinemia has recently been described as the only parameter being more frequently present in acromegalic cats compared to non-acromegalic diabetic cats (Niessen 2010). Previously, hyperphosphatemia (without azotemia) has been seen in a substantial percentage of acromegalic cats (Peterson et al 1990). There is some controversy as to which chronic renal failure develops more frequently in acromegalic cats.

\section{Diagnostic imaging}

Conventional radiography may reveal hepatomegaly, renomegaly, and rarely splenomegaly as well as cardiomegaly; pulmonary edema or pleural effusion may be seen if congestive heart failure is present. Additionally, diffuse increase in the oropharyngeal region, enlargement of the mandible, hyperostosis of the bony calvarium and nasal bones and degenerative arthropathy of the large joints may be seen.

Main ultrasonographic findings include hepatomegaly, increased kidney length, increased pancreatic thickness and bilateral adrenomegaly. Bilateral adrenomegaly with adrenal thickness was found in 54\% of acromegalic cats (Lourenço et al 2015). Adrenomegaly is not pathognomonic for HS, because it is also found in cats with hyperadrenocorticism, hyperthyroidism and hyperaldosteronism (Combes et al 2012). Adrenomegaly in conjunction with enlargement of other abdominal organs, in particular the kidneys should raise suspicion for HS.

Pituitary imaging plays a major role in the work-up of cats with suspected HS. CT/MRI studies should always include post-contrast images, because the mass may not be visible on native studies. In the majority of cats, the disease is caused by an adenoma of the somatotrophic cells. The masses can become quite large and suprasellar extension with involvement of the diencephalon is a frequent finding (Posch et al 2011). MRI with adequate resolution is superior to CT to detect small pituitary masses. In a recent study pituitary masses were identified in CT images in 64/68 cats with HS. The masses had dorsoventral dimensions between 4.2-16 mm (median 6.1). In 2/68 cats masses were only identified by means of MRI and in the other 2 cats acidophilic hyperplasia was found at necropsy (Lamb et al 2014). The same study demonstrated that $66 \%$ of cats had values for facial bone measurements and $19 \%$ of cats values for soft tissue measurements outside the normal range. 


\section{Hormonal evaluation}

Diagnosis requires the demonstration of elevated GH or elevated IGF-1 concentrations. GH in cats can be measured in heterologous canine and ovine radioimmunoassays. However, availability of the assay is limited and strict pre-analytical conditions have to be followed. Due to the episodic nature of $\mathrm{GH}$ secretion a single elevated $\mathrm{GH}$ concentration is not diagnostic and it is currently recommended to collect 3-5 samples for GH measurement at 10-minutesintervals (Meij et al 2010).

Measurement of IGF-1 has become a popular test for feline HS during the last years. It reflects the 24-hour GH secretion, its measurement has several advantages over the measurement of IGF-1: no pulsatile secretion requiring just a single random blood sample, conservation of structure across species and therefore measurement in assays designed for humans, serum sample can be sent by regular mail. However, IGF-1 is not $100 \%$ reliable for the diagnosis or the rule out of HS. IGF-1 can be normal in cats with HS due to the following causes: early stage of the acromegalic disease, prolonged starvation; lack of insulin (i.e. measurement performed prior to insulin therapy); interference with IGF-1 binding proteins in some assays. It is also likely, that the currently used cut-off of $>1000 \mathrm{ng} / \mathrm{ml}$ is too high to detect mild or early stages of the disease. Main causes for false positive IGF-1 results in cats without acromegaly are assay-related issues (e.g. incomplete removal of IGF-1-binding proteins in some assays), vigorous and/or long-term insulin therapy (Reusch 2015). The question, if IGF-1 is influenced by body weight is controversial.

\section{Treatment}

In some cats with HS, treatment is limited to treatment of the diabetes because the owner denies treatment of the acromegalic condition. Insulin resistance varies widely between acromegalic cats, in some cats, glycemic control may be achieved with "normal" insulin doses $(1-3 \mathrm{U} /$ cat BID), in others, much higher doses are required. We usually increase the insulin dose in steps of $0.5-1.0 \mathrm{U} /$ cat BID approximately every 5 to 7 days, until glycemic control is acceptable, e.g. most blood glucose levels throughout the day are between approximately $100-300 \mathrm{mg} / \mathrm{dl}(5.6-17.0 \mathrm{mmol} / \mathrm{l})$. Close monitoring of the cats is mandatory, and home-monitoring of glucose by the owners is highly recommended. Specific treatment modalities for HS include pituitary surgery, radiation therapy and medical therapy. Pituitary surgery and radiation therapy will be covered in other presentations.

In human medicine 3 classes of drugs are used for medical treatment of HS: somatostatin receptor ligands, dopamine agonists and GH receptor agonists. Until recently, somatostatin receptor ligands were considered to be of limited usefulness, most likely due to their selective binding to the somatostatin receptor subtypes (high for SSTR2, moderate for SSTR3 and 5, very low for SSTR 1 and 4). A recent study shows promising results by using the new somatostatin analog pasireotide with high affinity for SSRT 1, 2, 3, 5. BID application of $0.03 \mathrm{mg} / \mathrm{kg}$ pasireotide for 3 consecutive days in 12 diabetic cats with HS results in a significant decrease in IGF-1 and insulin dose. Adverse effects were small intestinal diarrhea in $3 / 12$ cats and biochemical hypoglycemia in $5 / 12$ cats (Scudder et al 2015). The same research group also evaluated Pasireotide Long-Acting Release, $8 \mathrm{mg} / \mathrm{kg}$ once per month for 6 months in 12 diabetic cats with acromegaly. 7/12 completed the study, in 3/7 cats diabetic remission was achieved. Adverse effects were soft stools in the majority of cats, worsening of polyphagia, hypoglycemia and delayed hair growth (Goestelow et al 2014, abstract). Further studies are needed to optimize the dose to enable higher success and lower withdrawal rates and to evaluate the long-term outcome in cats treated with pasireotide. Currently, pasireotide is a very expensive drug.

The dopamine agonist L-deprenyl was used for 9 months in one cat without improvement in clinical signs and insulin requirements (Abraham et al 2002). The use of $\mathrm{GH}$ receptor 
agonists has not been described in cats with HS.

\section{Prognosis}

If the disease is left untreated, the prognosis is usually guarded to poor. In many cats, clinical signs and insulin resistance are progressive, rendering insulin treatment increasingly difficult. Owner may become frustrated by the poor control of the diabetes and the increasing or fluctuating insulin requirement and may opt for euthanasia. Other cats are euthanized or die because of the development of congestive heart failure, renal failure, respiratory distress or neurological signs associated with expansion of the pituitary tumor. However, we have also seen cats in which HS is stable and diabetes sufficiently regulated for a fairly long time (1 $1 \frac{1}{2}$ years). Cats, in which the GH excess is treated with either pituitary surgery or radiation therapy, may have a favorable outcome. Glycemic control often improves and diabetic remission is possible, survival for several years has been seen.

\section{References}

1. Abraham LA, et al: Treatment of an acromegalic cat with dopamine agonist L-deprenyl, Aust Vet J 80:479, 2002.

2. Allgoewer I, et al: Somatotropes Hypophysenadenom mit Läsion des N. oculomotorius be einer Katze, Tierärztl Prax 26:267, 1998.

3. Berg RIM, et al: Serum insulin-like growth factor-I concentrations in cats with diabetes mellitus and acromegaly, J Vet Intern Med 21:892, 2007.

4. Capen CC: Endocrine Glands, In Maxie MG, editor: Jubb, Kennedy, and Palmer's Pathology of Domestic Animals, ed 5, Vol 3, 2007, Saunders Elsevier.

5. Combes A, et al: Ultrasonographic measurements of adrenal glands in cats with hyperthyroidism, Vet Radio \& Ultrasound 53(2):210, 2012.

6. Dirtu AC, Niessen SJM, Jorens PG, Covaci A: Organohalogenated contaminants and type 2 diabetes mellitus: a clue for endocrine disruption in humans? Environ Internat 57-58:60, 2013.

7. Eigenmann J, Wortman J, Haskins M.: Elevated growth hormone levels and diabetes mellitus in a cat with acromegalic features. J Am Anim Hosp Assoc 20:747, 1984.

8. Gembardt $\mathrm{C}$ and Loppnow H: Zur Pathogenese des spontanen Diabetes mellitus der Katze. II. Mitteilung: Azidophile Adenome des Hypophysenvorderlappens und Diabetes mellitus in zwei Fällen, Berl. Münch. Tierärztl. Wschr. 89:336, 1976.

9. Gostelow R, et al: Paisreotide (SOM230) long-acting release treatment for feline hypersomatotropism: a proof of concept trial, ACVIM Forum 2014, Nashville, abstract.

10. Heinrichs M, Baumgärtner W, Krug-Manntz S: Immunocytochemical demonstration of growth hormone in an acidophilic adenoma of the adenohypophysis in a cat, Vet Pathol 26:179, 1989.

11. Lamb CR, et al: Computed tomographic signs of acromegaly in 68 diabetic cats with hypersomatotropism, J Feline Med Surg 16(2):99, 2014.

12. Lichtensteiger CA, Wortman JA, Eigenmann JE: Functional pituitary acidophil adenoma in a cat with diabetes mellits and acromegalic features, Vet Pathol 23:518, 1986.

13. Lopes MBS: Growth hormone-secreting adenomas: pathology and cell biology, Neurosurg Focus 29:1, 2010.

14. Lourenço BN, Randall E, Seiler G, Lunn KF: Abdominal ultrasonographic findings in acromegalic cats, J Feline Med Surg 17(8):698, 2015.

15. Meij BP, van der Vlugt-Meijer, van den Ingh TSGAM, Rijnberk A: Somatotroph and corticotroph pituitary adenoma (double adenoma) in a cat with diabetes mellitus and hyperadrenocorticism, J Comp Path 130:209, 2004.

16. Meij BP, Kooistra HS, Rijnberk A: Hypothalamus-pituitary system. In Rijnberk A, Kooistra HS, editors: Clinical Endocrinology of Dogs and Cats, ed 2, Hannover, 2010, Schlütersche.

17. Middleton DJ, Culvenor JA, Vasak E, Mintohadi K: Growth hormone-producing pituitary adenoma, elevated serum somatomedin C concentration and diabetes mellitus in a cat, Can Vet J 26:169, 1985.

18. Morrison SA, Randolph J, Lothrop CD: Hypersomatotropism and insulin-resistant diabetes mellitus in a cat, J Am Vet Med Assoc 194:91, 1989.

19. Myers JA, Lunn KF, Bright JM: Echocardiographic findings in 11 cats with acromegaly, J Vet Intern Med 28(4), 2014. 
20. Niessen SJM, et al: Feline acromegaly: an underdiagnosed endocrinopathy? J Vet Intern Med 21:899, 2007.

21. Niessen S: Feline acromegaly, an essential differential diagnosis for the difficult diabetic, J Feline Med Surg 12:15, 2010.

22. Niessen SJM, et al: Studying cat (felis catus) diabetes: beware of the acromegalic imposter, PLoS ONE 10(5):1, 2015.

23. Norman EJ, Mooney CT: Diagnosis and management of diabetes mellitus in five cats with somatotrophic abnormalities, J Feline Med Surg 1:183, 2000.

24. Peterson ME, et al: Acromegaly in 14 cats, J Vet Intern Med 4:192, 1990.

25. Posch B, Dobson J, Herrtage M: Magnetic resonance imaging findings in 15 acromegalic cats, Vet Radiol Ultrasound 52:422, 2011.

26. Resmini E, Minuto F, Colao A, Ferone D: Secondary diabetes associated with principal endocrinopathies: the impact of new treatment modalities, Acta Diabetol 46:85, 2009.

27. Reusch CE: Disorders of Growth Hormone In: Feldman EC, Nelson RW, Reusch CE, Scott Moncrieff JCR, Behrend EN (eds). Canine \& Feline Endocrinology. St. Louis, Missouri: Elsevier, 2015, pp. 37-76.

28. Schäfer S, et al: Evaluation of Insulin-like growth factor-1 (IGF-1), total thyroxine (TT4), feline pancreasspecific lipase (fPLI), and urinary corticoid-to-creatinine ratio (UCCR) in cats with diabetes mellitus in Switzerland and The Netherlands, 2016, submitted.

29. Sharman M, FitzGerald L, Kiupel M: Concurrent somatotroph and plurihormonal pituitary adenomas in a cat, J Feline Med Surg, accepted, 2013. 